\title{
Induction of aneurysmogenic high positive wall shear stress gradient by wide angle at cerebral bifurcations, independent of flow rate
}

\author{
Alexandra Lauric, PhD, ${ }^{1,2}$ James E. Hippelheuser, BSc, ${ }^{1}$ and Adel M. Malek, MD, PhD1,2 \\ ${ }^{1}$ Cerebrovascular and Endovascular Division, Department of Neurosurgery, Tufts Medical Center; and ${ }^{2}$ Tufts University School of \\ Medicine, Boston, Massachusetts
}

OBJECTIVE Endothelium adapts to wall shear stress (WSS) and is functionally sensitive to positive (aneurysmogenic) and negative (protective) spatial WSS gradients (WSSG) in regions of accelerating and decelerating flow, respectively. Positive WSSG causes endothelial migration, apoptosis, and aneurysmal extracellular remodeling. Given the association of wide branching angles with aneurysm presence, the authors evaluated the effect of bifurcation geometry on local apical hemodynamics.

METHODS Computational fluid dynamics simulations were performed on parametric bifurcation models with increasing angles having: 1) symmetrical geometry (bifurcation angle $60^{\circ}-180^{\circ}$ ), 2) asymmetrical geometry (daughter angles $30^{\circ} / 60^{\circ}$ and $30^{\circ}\left(90^{\circ}\right.$ ), and 3) curved parent vessel (bifurcation angles $60^{\circ}-120^{\circ}$ ), all at baseline and double flow rate. Time-dependent and time-averaged apical WSS and WSSG were analyzed. Results were validated on patient-derived models.

RESULTS Narrow symmetrical bifurcations are characterized by protective negative apical WSSG, with a switch to aneurysmogenic WSSG occurring at angles $\geq 85^{\circ}$. Asymmetrical bifurcations develop positive WSSG on the more obtuse daughter branch. A curved parent vessel leads to positive apical WSSG on the side corresponding to the outer curve. All simulations revealed wider apical area coverage by higher WSS and positive WSSG magnitudes, with increased bifurcation angle and higher flow rate. Flow rate did not affect the angle threshold of $85^{\circ}$, past which positive WSSG occurs. In curved models, high flow displaced the impingement area away from the apex, in a dynamic fashion and in an angledependent manner.

CONCLUSIONS Apical shear forces and spatial gradients are highly dependent on bifurcation and inflow vessel geometry. The development of aneurysmogenic positive WSSG as a function of angular geometry provides a mechanotransductive link for the association of wide bifurcations and aneurysm development. These results suggest therapeutic strategies aimed at altering underlying unfavorable geometry and deciphering the molecular endothelial response to shear gradients in a bid to disrupt the associated aneurysmal degeneration.

https://thejns.org/doi/abs/10.3171/2018.3.JNS173128

KEYWORDS intracranial aneurysms; bifurcation apex; wall shear stress; vascular disorders

I NTRACRANIAL aneurysms are pathological dilatations of the arterial wall, which often occur in association with predisposing arterial geometries, such as bifurcations and curved vessels. ${ }^{11}$ The interaction between biological and hemodynamic forces is believed to be critical to the pathogenesis of aneurysm initiation, growth, and rupture. ${ }^{23}$ In particular, wall shear stress (WSS) has been determined to impact the mechanics of the arterial wall and to be a critical mediator of gene expression regulation via endothelial mechanoreceptors..$^{20}$ Arterial bifurcations are characterized hemodynamically by particular flow patterns identified as disturbed laminar shear stress. ${ }^{19} \mathrm{Re}-$ gions of disturbed flow display high temporal and spatial gradients, which were shown to trigger dramatic changes in endothelial cell (EC) shape, density, and rate of division. ${ }^{4,19}$ The capacity of the ECs to sense and adapt to WSS

ABBREVIATIONS BA = basilar artery; $\mathrm{CFD}=$ computational fluid dynamics; $\mathrm{EC}=$ endothelial cell; $\mathrm{MCA}=$ middle cerebral artery; TAVel = time-averaged velocity; TAWSS = time-averaged wall shear stress; TAWSSG = TAWSS gradient; WSS = wall shear stress; WSSG = WSS gradient.

SUBMITTED December 13, 2017. ACCEPTED March 1, 2018.

INCLUDE WHEN CITING Published online August 10, 2018; DOI: 10.3171/2018.3.JNS173128. 
variations, but also to discriminate between positive and negative spatial WSS gradients (WSSGs) was previously documented. $5,6,24$

Positive spatial WSSG occurs when WSS is increasing with direction of flow, such as in flow acceleration, whereas negative WSSG is seen in cases of decelerating flow. Destructive cellular and extracellular responses to high WSS have been shown to be triggered by positive WSSG and mitigated by negative WSSG. ${ }^{5,6,24}$ Positive WSSG also leads to endothelial misalignment and turnover, with high proliferation and apoptosis, ${ }^{6}$ whereas negative WSSG leads to an opposite, protective effect. Surgically created de novo branching points showed that changes in arterial morphology resulted in altered WSS patterns, and triggered arterial remodeling resembling early-stage intracranial aneurysms in areas of flow acceleration and positive WSSG, including a decrease in the overall thickness of the media and intima, loss of the internal elastic lamina, and disrupted endothelium. ${ }^{17}$

Aneurysm presence at cerebral bifurcations was recently shown to be associated with wider bifurcation angles in both middle cerebral and basilar bifurcations. ${ }^{1,21,25}$ Basilar bifurcations may widen with age, more so in women, possibly triggering tissue degeneration due to changes in the local hemodynamic environment. ${ }^{25}$

Although the mechanism for aneurysm initiation is not completely understood, consensus is building regarding the disruptive role of local high WSS and high positive WSSG in the pathological process of aneurysm origination. $., 6,17,24$ Studies in animals subjected to unilateral or bilateral common carotid artery ligation have shown that, without other known predisposing factors, flow increase alone at cerebral bifurcations can lead to EC remodeling consistent with aneurysm initiation. ${ }^{10,18}$ We hypothesized that WSSG direction and magnitude are dependent on bifurcation geometry, and we sought to explore the effect of individual daughter branch angulations, mother vessel geometry, and flow rate on spatial shear gradient direction in order to link known aneurysmogenic shear conditions to observed aneurysm colocation in wider bifurcations.

\section{Methods}

\section{Parametric Models}

Three series of parametric models of the middle cerebral artery (MCA) bifurcation were constructed to account for the morphological configurations observed in patient data sets with respect to bifurcation angles and parent vessel geometry (Fig. 1A). All models were created using SolidWorks as previously described..$^{12,14,25}$ This research was approved by the Tufts Health Sciences Campus Institutional Review Board.

The first set of parametric models was created with a straight parent vessel that had a length of $15 \mathrm{~mm}$ and a radius of $1.3 \mathrm{~mm}$. Symmetrical daughter vessels (length $10 \mathrm{~mm}$, radius $1.032 \mathrm{~mm}$ ) were joined to the inflow vessel, such that the combination of the radii for the parent and daughter vessels followed the vascular optimality principle. ${ }^{21}$ Five bifurcation models were created with total bifurcation angles of $60^{\circ}, 80^{\circ}, 85^{\circ}, 120^{\circ}$, and $180^{\circ}$ (Fig. 1B).

A second set of asymmetrical parametric models was created starting from the $60^{\circ}$ symmetrical model and increasing only one of the angles formed between the mother and daughter vessels. Two additional bifurcation models were created with total bifurcation angles of $90^{\circ}$ and $120^{\circ}$ (Fig. 1C).

Curved mother vessel morphologies were created starting from the straight symmetrical models by adding a proximal $90^{\circ}$ bend with a radius of curvature of $5 \mathrm{~mm}$ along the vessel centerline. The resulting inflow vessel length was the same as that of the straight models (Fig. 1D). Curved models were created with symmetrical bifurcation angles of $60^{\circ}, 80^{\circ}$, and $120^{\circ}$.

\section{Cerebral Angiography and Data Processing}

Three-dimensional cerebral angiograms were obtained using Siemens Artis biplane systems in 3 patients harboring MCA bifurcation aneurysms, and the lesions were reconstructed using the embedded Leonardo clinical software package to yield a 3D volume. Volumetric data sets, including aneurysm and parent vessel, were analyzed in Amira version 5.4 (FEI) for segmentation in 3D space.

\section{Patient-Derived Models}

Following vessel segmentation, the aneurysms were digitally removed by local Laplacian smoothing, ${ }^{3}$ and the parent vessels were reconstructed in MeshLab version 1.3.1 (ISTI-CNR). Laplacian filtering tends to shrink the surface being smoothed. A high number of smoothing iterations was applied on an area constrained to the aneurysm dome, which resulted in the removal of the aneurysm while preserving the surrounding vasculature. The MCA segment proximal to the site of the aneurysm was kept sufficiently long (at least 5 times the diameter of the parent vessel) to enable the laminar flow to become fully developed and avoid entrance effects.

\section{Computational Fluid Dynamics}

Computational fluid dynamics (CFD) simulations were performed using Star-CCM+ (CD-Adapco), by modeling laminar transient flow with a Carreau non-Newtonian profile to better approximate the viscosity and flow of blood.22 For each model, the velocity magnitude was scaled to an idealized mother vessel WSS of approximately 15 dynes/ $\mathrm{cm}^{2}$ at the straight vessel inlet. ${ }^{16}$ Pulsatile flow was applied at the inlet for 3 complete cardiac cycles to allow flow to develop adequately, and the third cycle was used for analysis. Each cycle period was $\mathrm{T}=1$ second and had a time step of $\mathrm{t}=0.002$ second, with the peak systole at $\mathrm{t}=0.16$ second. At the outlets, gauge pressure was set to $0 \mathrm{~mm} \mathrm{Hg}$, as previously reported. ${ }^{2}$ The effect of higher velocity on the bifurcation hemodynamics was simulated by doubling the velocity magnitude along the input velocity profile, resulting in WSS of 27.5 dynes $/ \mathrm{cm}^{2}$ at the inlet.

Postprocessing analysis was performed using EnSight 10.0 (Computational Engineering International). Consistent with previous research, $, 13,17,18$ WSS and spatial WSSG were evaluated at each time step as well as time averaged (TAWSS and TAWSSG) along a longitudinal cut covering the apex of the bifurcation and extending downstream. Because WSS and WSSG are surface parameters, mea- 


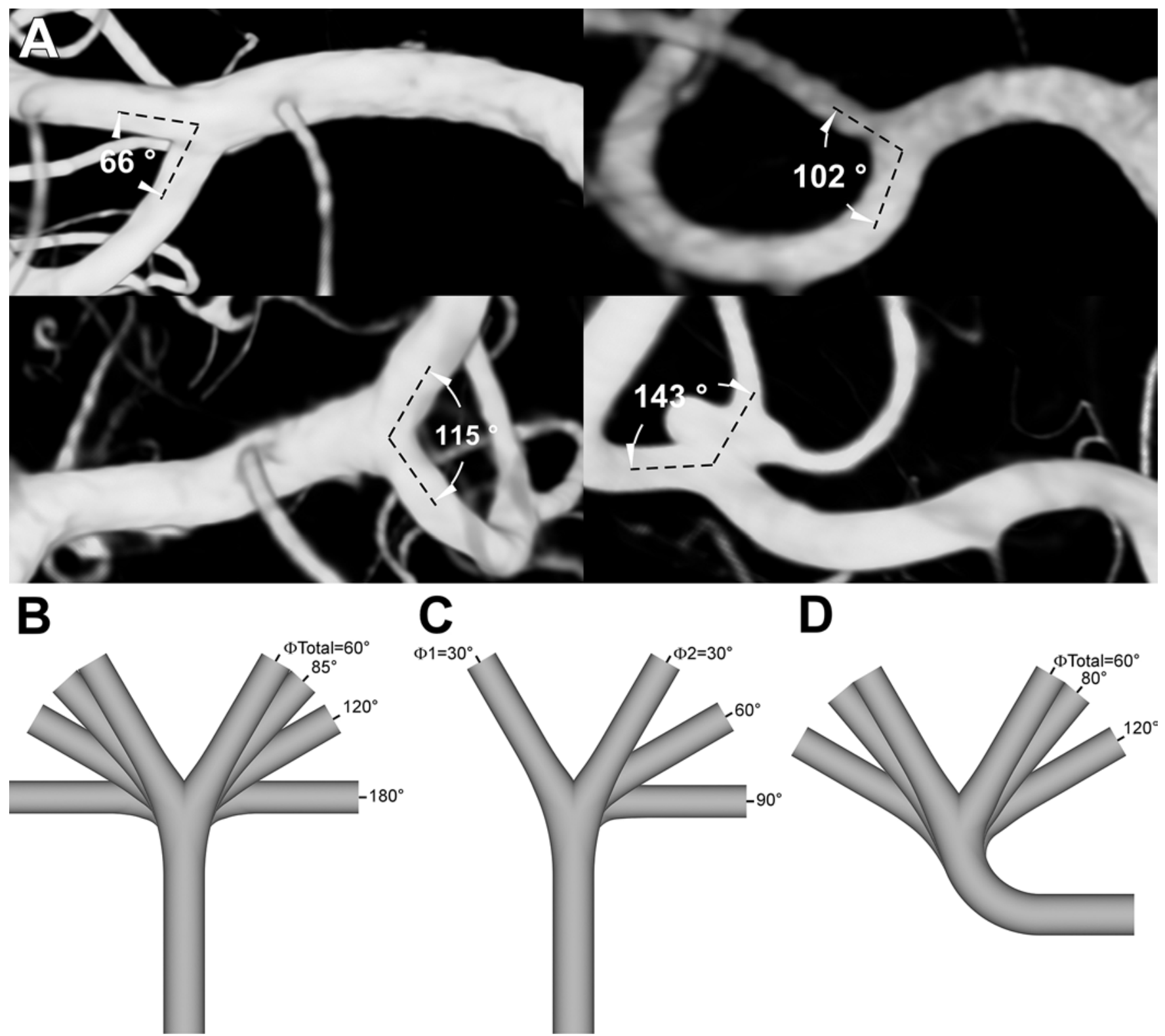

FIG. 1. A: Volumetric representations of 3D rotation angiography showing anatomical variations in the MCA bifurcations. B: Symmetrical parametric models (total bifurcation angles $\Phi_{1}+\Phi_{2}=60^{\circ}, 80^{\circ}$ (not shown), $85^{\circ}, 120^{\circ}$, and $180^{\circ}$ ). C: Asymmetrical parametric models (fixed $\Phi_{1}=30^{\circ}$ angle, and varying $\Phi_{2}=30^{\circ}, 60^{\circ}$, and $90^{\circ}$ ). D: Curved parent vessel morphology with symmetrical bifurcation modeling (total bifurcation angles $\Phi_{1}+\Phi_{2}=60^{\circ}, 80^{\circ}$, and $120^{\circ}$ ).

sured exclusively on the vessel wall, the result of this longitudinal cut was a 1D line, positioned on the 3D model and passing longitudinally through the apex. WSSG was positive in areas of flow acceleration along the wall (increasing WSS) and negative in areas of flow deceleration (decreasing WSS). ${ }^{18}$

\section{Results}

\section{Parametric Models}

CFD analysis on symmetrical models showed that the impingement area of narrow bifurcations $\left(60^{\circ}\right.$ and $\left.80^{\circ}\right)$ is characterized by high TAWSS $\left(>30\right.$ dynes $/ \mathrm{cm}^{2}$ ) focused exclusively at the apex. These regions are also character- ized by high negative TAWSSG $\left(<-30\right.$ dynes $\left./\left(\mathrm{cm}^{2} \mathrm{~mm}\right)\right)$. A threshold angle of $85^{\circ}$ was identified as the value above which WSSG at the apex switches from negative (protective) to positive (aneurysmogenic) values. Still, as shown in Fig. 2, at $85^{\circ}$ the positive WSSG peak is narrowly located at the bifurcation apex in the region of the apical intimal pad, which is known to have a protected collagen band. Further increase of the total bifurcation angle past $85^{\circ}$ induces a widening of the area covered by aneurysmogenic positive WSSG and high WSS. The flow impingement areas of wider bifurcation angles are characterized by lower WSS flanked by distal WSS peaks. The area between these WSS peaks is characterized by aneurysmogenic high positive WSSG. 


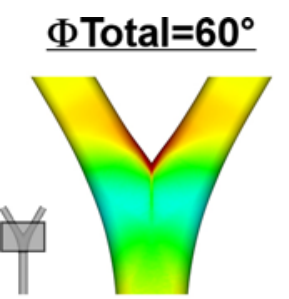

A

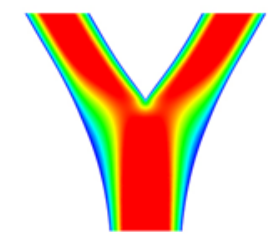

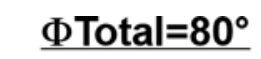

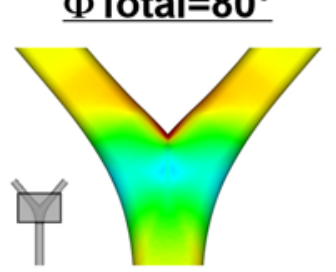

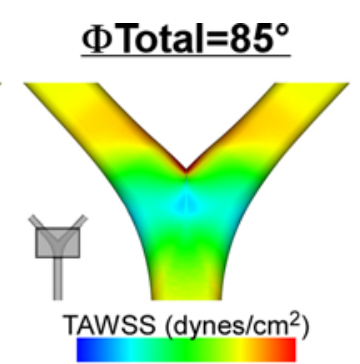

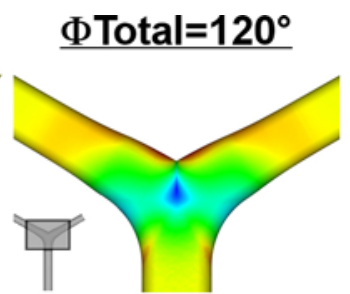

$\Phi$ Total $=180^{\circ}$

$0.0 \quad 10.0 \quad 20.0$

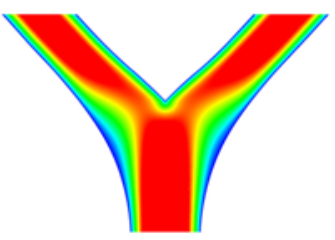

TAVel $(\mathrm{m} / \mathrm{s})$
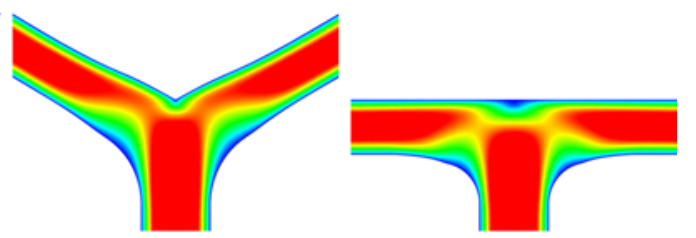

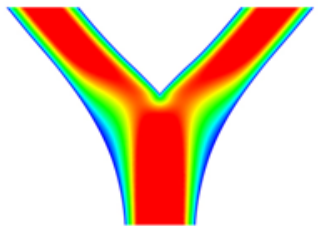

B

$0.00 \quad 0.05 \quad 0.10$
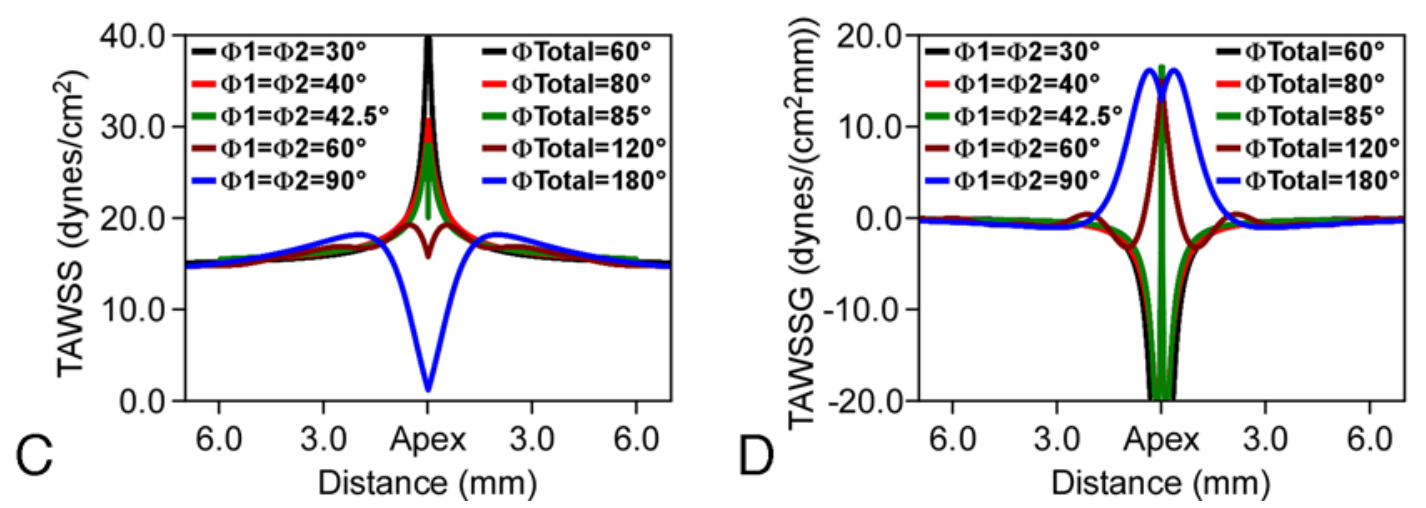

FIG. 2. CFD analysis on symmetrical models. A: TAWSS on 3D models. B: Time-averaged velocity (TAVel) shown on the longitudinal cut through the 3D volume. C: Plot of TAWSS on longitudinal 1D cut at the apex. D: Plot of TAWSSG on longitudinal 1D cut at the apex. Positive TAWSSG is detected starting from a bifurcation angle of $85^{\circ}$. Figure is available in color online only.

CFD simulations on asymmetrical models (Fig. 3) showed that the bifurcation apex is characterized by high TAWSS and negative TAWSSG (protective) on the side corresponding to the smaller angle $\left(30^{\circ}\right)$, and by lower WSS and high positive WSSG (aneurysmogenic) on the side corresponding to the higher angle. Whereas on the side corresponding to the smaller angle the area of high WSS is focused at the apex, on the side corresponding to the wider angle $\left(60^{\circ}\right.$ and $\left.90^{\circ}\right)$ the area of aneurysmogenic positive WSSG widens with the size of the bifurcation angle onto the corresponding daughter vessel branch.

As shown in Fig. 4, modeling a curved inflow vessel resulted in the symmetrical bifurcation behaving similarly to an asymmetrical bifurcation. The bifurcation apex was characterized by sharply elevated TAWSS and negative (protective) TAWSSG on the side corresponding to the parent vessel inner curve, whereas the side of the bifurcation corresponding to the parent vessel outer curve (larger lateral angle) was subjected to high positive (aneurysmogenic) WSSG concentrated at the apex and dissipating distally into the daughter branch. This region subjected to high positive WSSG is very narrow at smaller bifurcation angles, but widens to as much as $3 \mathrm{~mm}$ distal from the apex for larger bifurcations.
Time-dependent flow analysis (Fig. 5) showed that for straight inlet models (Fig. 5A and B) the impingement region remains the same throughout the cardiac cycle. Whereas the magnitude of WSS and WSSG change at different time points, the overall distribution shape is maintained. In contrast, in curved models (Fig. 5C-E) the impingement regions shift during the cardiac cycle and migrate toward the side of the bifurcation corresponding to the parent vessel outer curve. This displacement, resulting from the centripetal force moving the flow along the circle of curvature of the inflow vessel, is more pronounced at peak systole and increases with larger bifurcation angles.

As expected, increasing the flow rate resulted in higher WSS and WSSG magnitude values in all models. However, use of a higher flow rate did not result in significant changes in the shape of the WSS and WSSG distributions (Fig. 6). For the straight symmetrical model of $80^{\circ}$, the apex is subjected to high WSS and high negative WSSG concentrated at the apex (Fig. 6A). Although a higher flow rate resulted in a wider bifurcation area (distal into the daughter branches) covered by higher WSS values, it did not lower the bifurcation angle threshold (identified as $85^{\circ}$ ) at which WSSG changes from negative to positive values. For the straight asymmetrical model $\left(30^{\circ}\right.$ and $60^{\circ}$ 
$\Phi 1=30^{\circ}, \Phi 2=30^{\circ}$

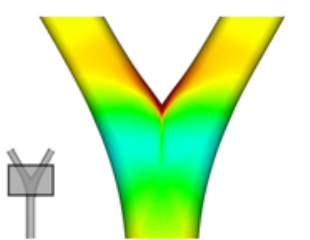

A

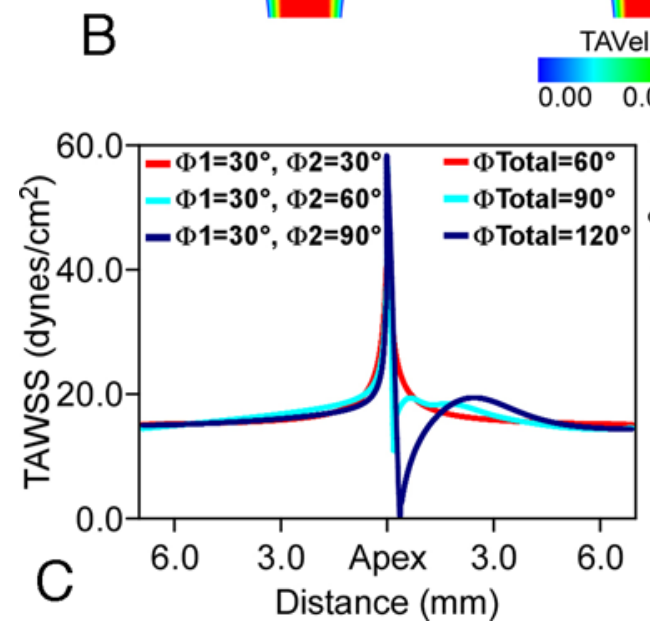

$\Phi 1=30^{\circ}, \Phi 2=60^{\circ}$

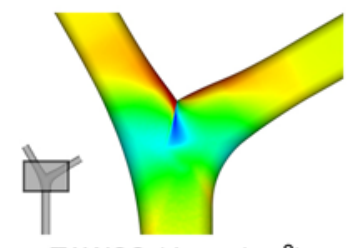

TAWSS (dynes $/ \mathrm{cm}^{2}$ )
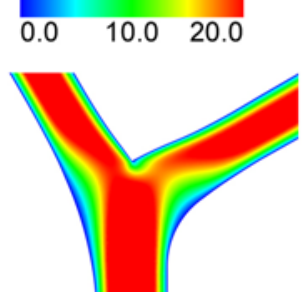

Vel $(\mathrm{m} / \mathrm{s})$
$\Phi 1=30^{\circ}, \Phi 2=90^{\circ}$
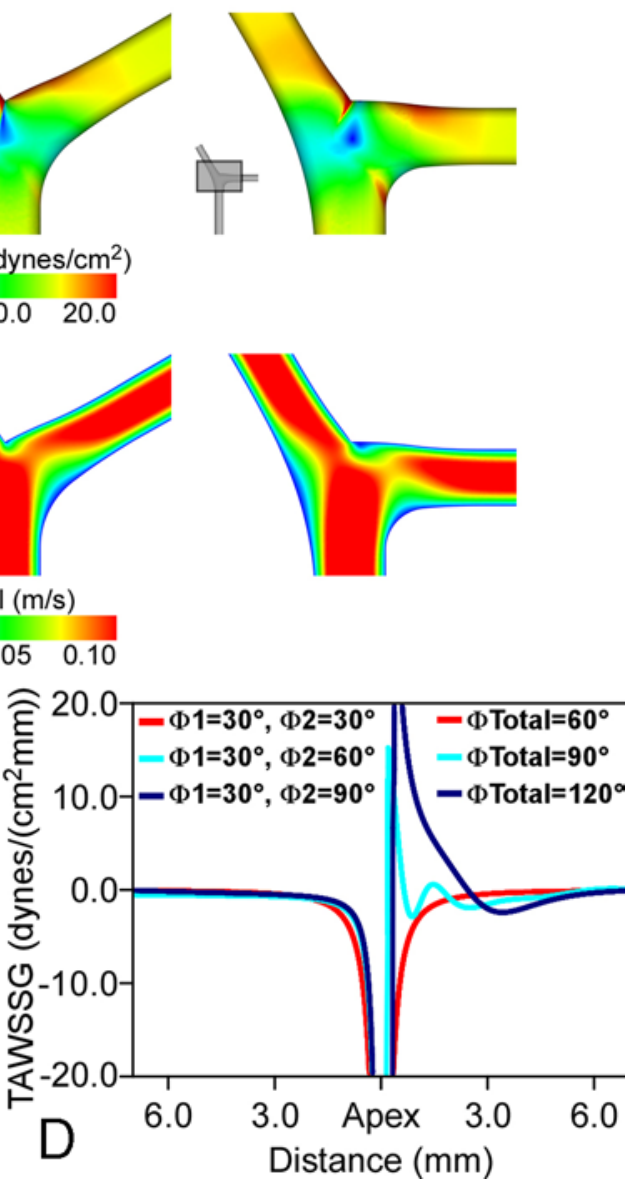

FIG. 3. CFD analysis on asymmetrical models. A: TAWSS on 3D models. B: TAVel shown on the longitudinal cut through the 3D volume. C: Plot of TAWSS on longitudinal 1D cut at the apex. D: Plot of TAWSSG on longitudinal 1D cut at the apex. The side corresponding to the wider angle is characterized by positive TAWSSG. Figure is available in color online only.

branch angles), higher flow resulted in a wider area of high WSS magnitude; however, the area subjected to high positive WSSG (corresponding the $60^{\circ}$ angle) remained the same, at approximately $0.5 \mathrm{~mm}$ from the apex (Fig. 6B). In curved models, higher flow simulation resulted in an increased displacement of the impingement regions (Fig. $6 \mathrm{C}-\mathrm{E})$. The displacement is noticeable during the entire cardiac cycle. In the $120^{\circ}$ curved model, the displacement at peak systole increased from $0.2 \mathrm{~mm}$ at normal flow to $0.4 \mathrm{~mm}$ under higher flow conditions.

\section{Patient-Derived Models}

Analysis of patient-derived models showed the bifurcation area being characterized by TAWSS peaks on each side of the apex, distally along the daughter branches (Fig. 7). This results in a wide area covered by high positive TAWSSG. These results are consistent with the results obtained from the parametric models.

\section{Discussion}

The interaction between vessel wall biology and hemodynamic forces is considered central to the pathogenesis of aneurysms, because the formation of aneurysms is believed to be related to destructive EC responses to high WSS. Vascular ECs prove to be particularly responsive to fluid shear stresses in regard to alignment, proliferation, apoptosis, and density. ${ }^{23}$ Whereas the capacity of the ECs to sense high WSS was previously established, more recent studies suggest that ECs are also sensitive to WSSGs and can distinguish between negative and positive spatial gradients. 5,6,24 WSSGs seem to either ameliorate or exacerbate high WSS responses. ${ }^{6}$ Negative WSSG suppresses EC proliferation and apoptosis, and promotes cell alignment in the direction of the flow. ${ }^{5,6} \mathrm{EC}$ response to high WSS and negative WSSG was found to be similar to that of ECs exposed to lower WSS. In contrast, high WSS in the presence of positive WSSG stimulates EC proliferation and apoptosis, and inhibits flow-induced EC alignment. ${ }^{5,6} \mathrm{ECs}$ exposed to high WSS and positive WSSG were found to migrate downstream away from the impingement area and the high WSSG region. ${ }^{24}$ This response requires fragmentation of the internal elastic lamina, which is facilitated by proteolytic enzymes degrading extracellular matrix components and altering the arterial structure, leading to aneurysmogenic changes by allowing the vessel to expand. ${ }^{5}$ 
$\Phi$ Total $=60^{\circ}$

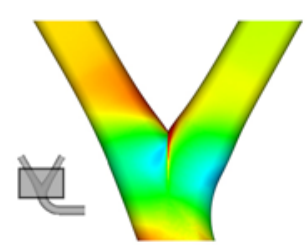

A

B

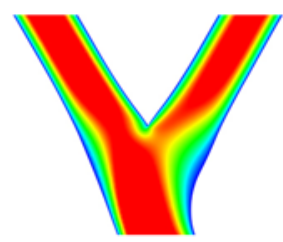

ФTotal $=80^{\circ}$
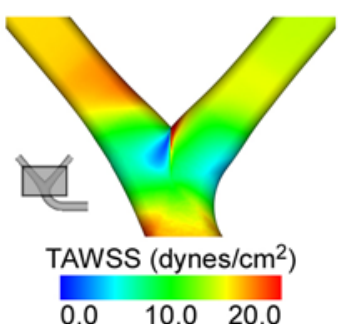

$0.0 \quad 10.0 \quad 20.0$

\section{$\Phi$ Total $=120^{\circ}$}
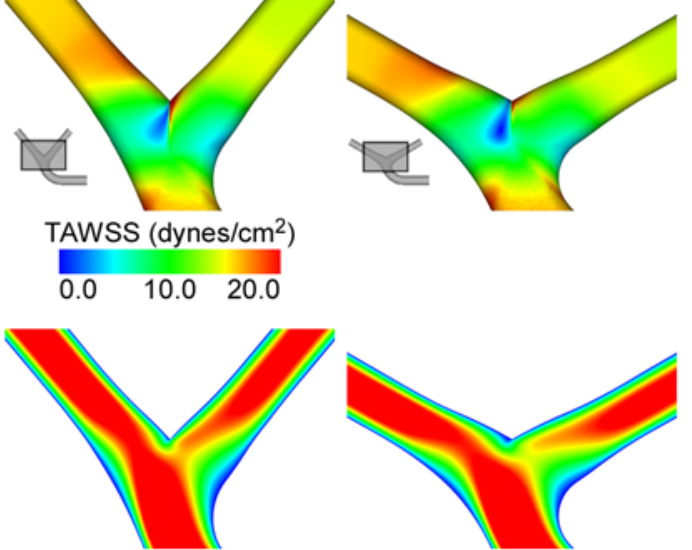

TAVel $(\mathrm{m} / \mathrm{s})$
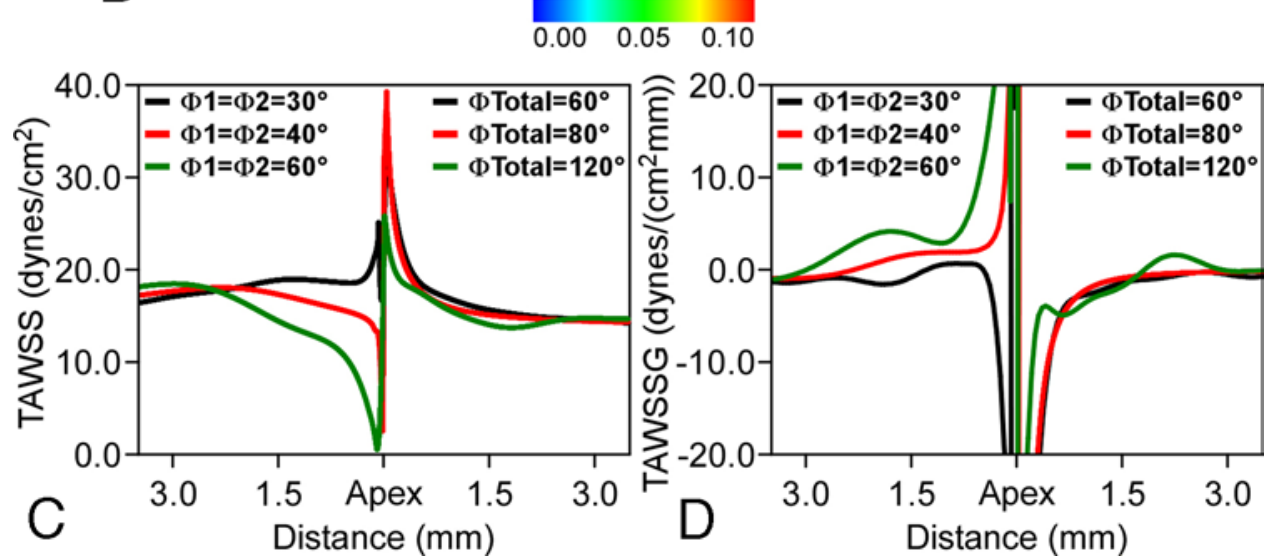

FIG. 4. CFD analysis on 3D curved models. A: TAWSS on 3D models. B: TAVel shown on the longitudinal cut through the 3D volume. C: Plot of TAWSS on longitudinal 1D cut at the apex. D: Plot of TAWSSG on longitudinal 1D cut at the apex. The side corresponding to the outer curve (the larger lateral angle) is characterized by positive TAWSSG. Figure is available in color online only.

EC responses to hemodynamic forces are particularly important at cerebral bifurcations, which are preferred locations for aneurysm formation. The impingement of the flow jet against the bifurcation apex produces a local elevation of WSS that was shown to be dependent on the bifurcation morphology. However, narrow bifurcations seem to be protected against aneurysmogenic changes, by mechanisms that are not completely understood.

The current study explored the effect of bifurcation geometry (especially the size of the bifurcation angle but also the parent vessel geometry) on local shear forces and the associated gradients. The rationale for this study is grounded in previously reported findings, showing that bifurcations harboring aneurysms are significantly wider compared to control nonaneurysmal bifurcations. ${ }^{1,25}$ In a large study of 400 patients, it was reported that those with basilar artery (BA) aneurysms had significantly wider BA bifurcations compared to healthy controls. ${ }^{25}$ Similarly, in a study of 146 MCA bifurcations, ${ }^{1}$ we found aneurysmharboring MCA bifurcations to be significantly wider compared to the bifurcations in healthy controls. MCA bifurcations tended to be more asymmetrical and the angle $\Phi_{2}$ (which was associated with the smaller daughter vessel) was reportedly wider than $\Phi_{1}$ (Fig. 1). In addition, 68\% of the aneurysms originated off the daughter vessel associated with the wider side angle $\left(\Phi_{2}\right)$, supporting the importance of asymmetrical bifurcation analysis. Whereas BA and MCA are relatively straight vessels, other intracranial arteries such as the internal carotid artery have been shown to have significant curvature variations along their length. ${ }^{15}$

Our results showed that the presence of aneurysmogenic positive WSSG at the bifurcation apex is determined by the angle of the bifurcation as well as the geometry of the parent vessel. Symmetrical bifurcations display negative WSSG up to a total bifurcation angle of $85^{\circ}$, and aneurysmogenic positive WSSG thereafter. With an increase in bifurcation angle, the bifurcation area subjected to high positive WSSG widens downstream onto the daughter branches. It is interesting that previous research on large patient data sets of $\mathrm{MCA}^{1}$ and BA aneurysms ${ }^{25}$ showed that no MCA aneurysm occurred at a bifurcation angle smaller than $121^{\circ}$ and no BA aneurysm occurred at a bifurcation angle smaller than $103^{\circ}$. Both values are above the $85^{\circ}$ threshold suggested by our results. It is plausible that a larger area of aneurysmogenic positive WSSG needs to be active away from the protective apical intimal padsuch as with angles larger than $120^{\circ}$-for the bifurcation 


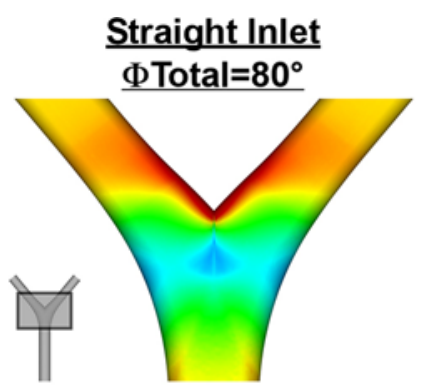

A

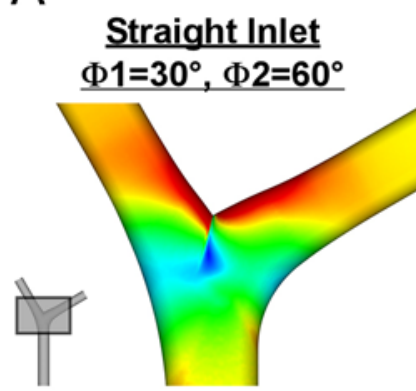

$\mathrm{B}$

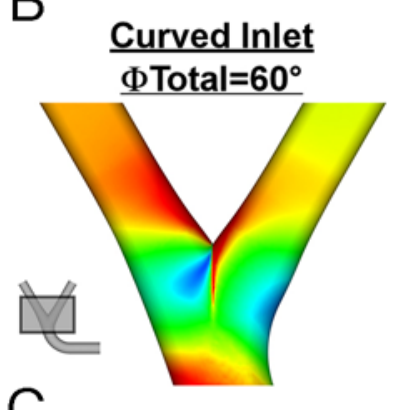

C

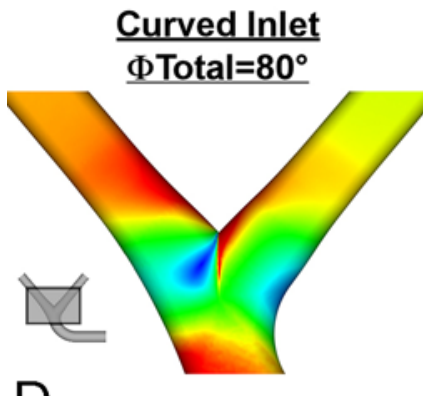

D

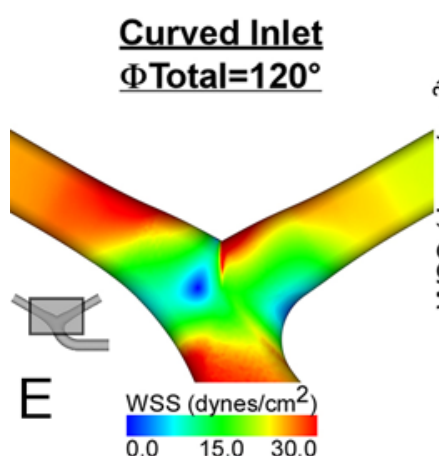

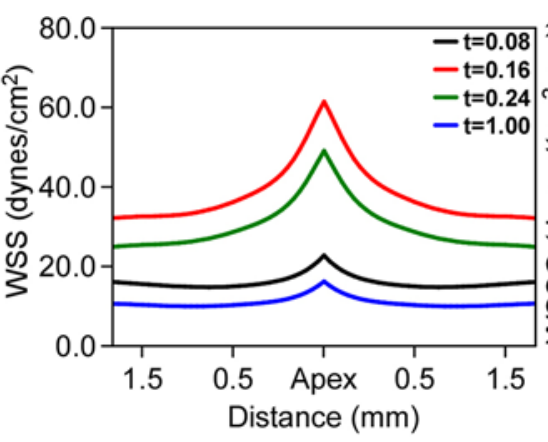
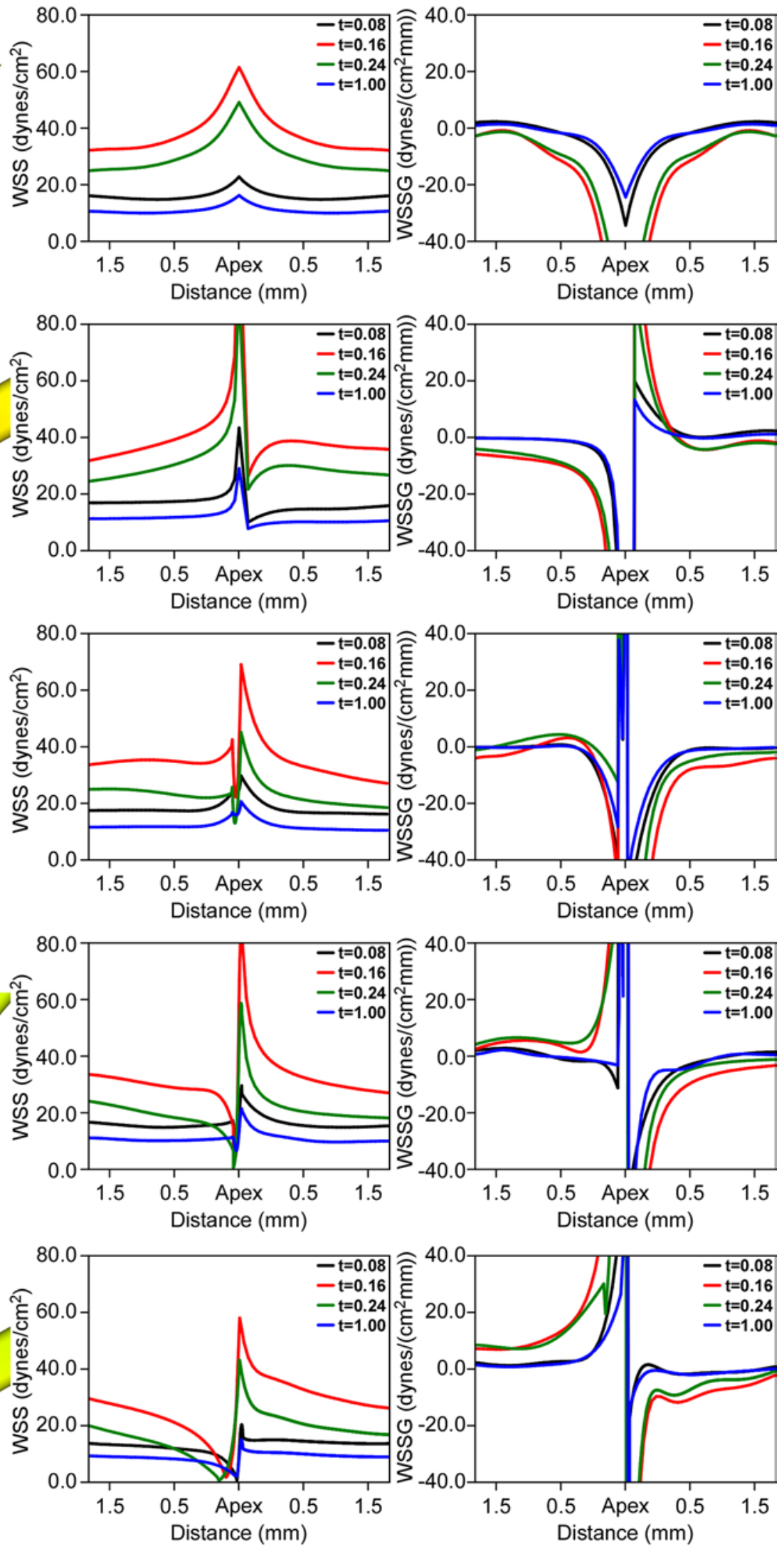

FIG. 5. Time-dependent analysis of WSS and WSSGs at peak systole (time step of $t=0.16)$. A: Symmetrical bifurcation. B: Asymmetrical bifurcation. C: Curved inlet and $60^{\circ}$ bifurcation angle. D: Curved inlet and $80^{\circ}$ bifurcation angle. E: Curved inlet and $120^{\circ}$ bifurcation angle. Figure is available in color online only. 

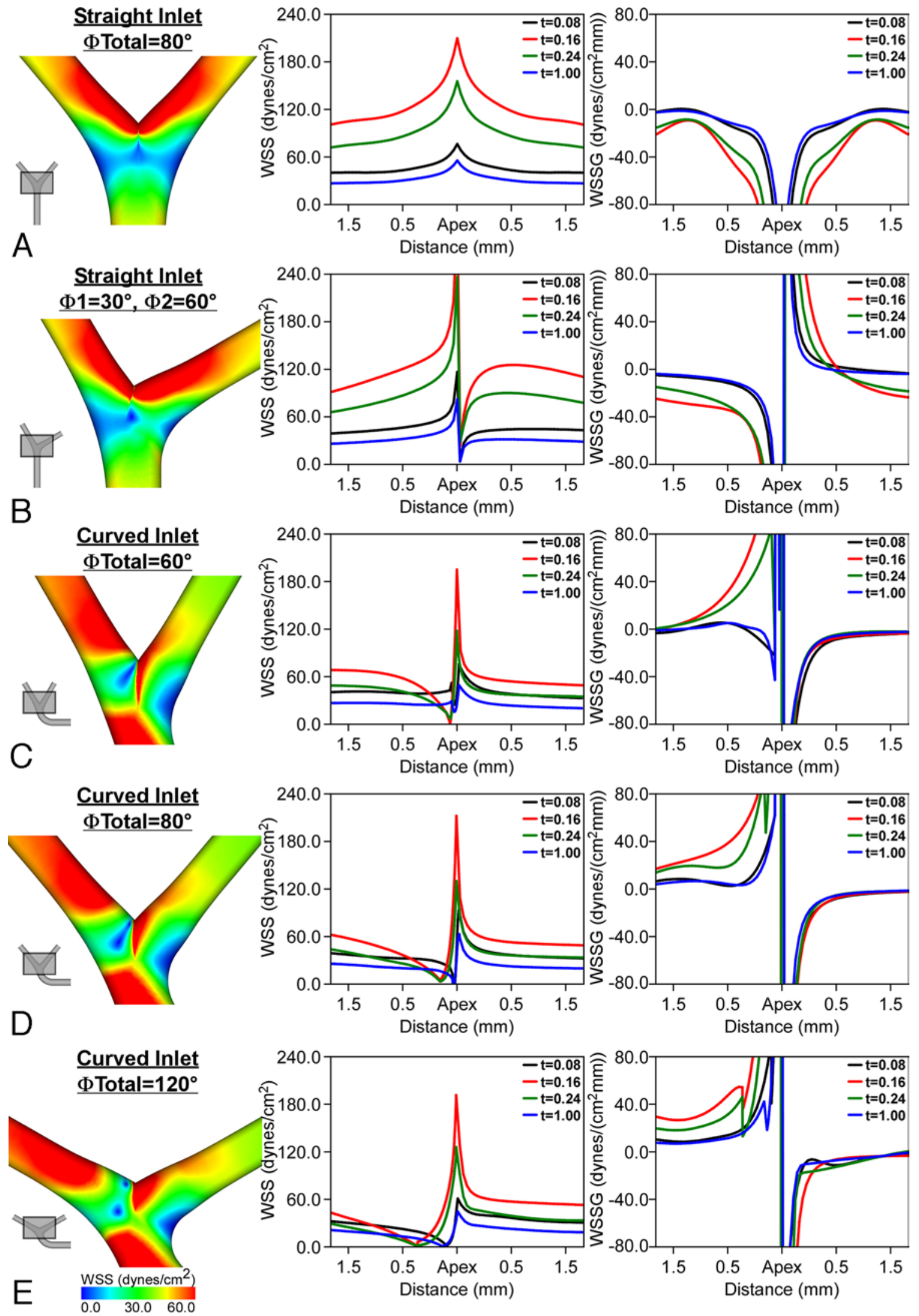

FIG. 6. Simulation of increased flow rate. Analysis of WSS and WSSGs at peak systole (time step of $t=0.16)$. A: Symmetrical bifurcation. B: Asymmetrical bifurcation. C: Curved inlet and $60^{\circ}$ bifurcation angle. D: Curved inlet and $80^{\circ}$ bifurcation angle. E: Curved inlet and $120^{\circ}$ bifurcation angle. Figure is available in color online only. 

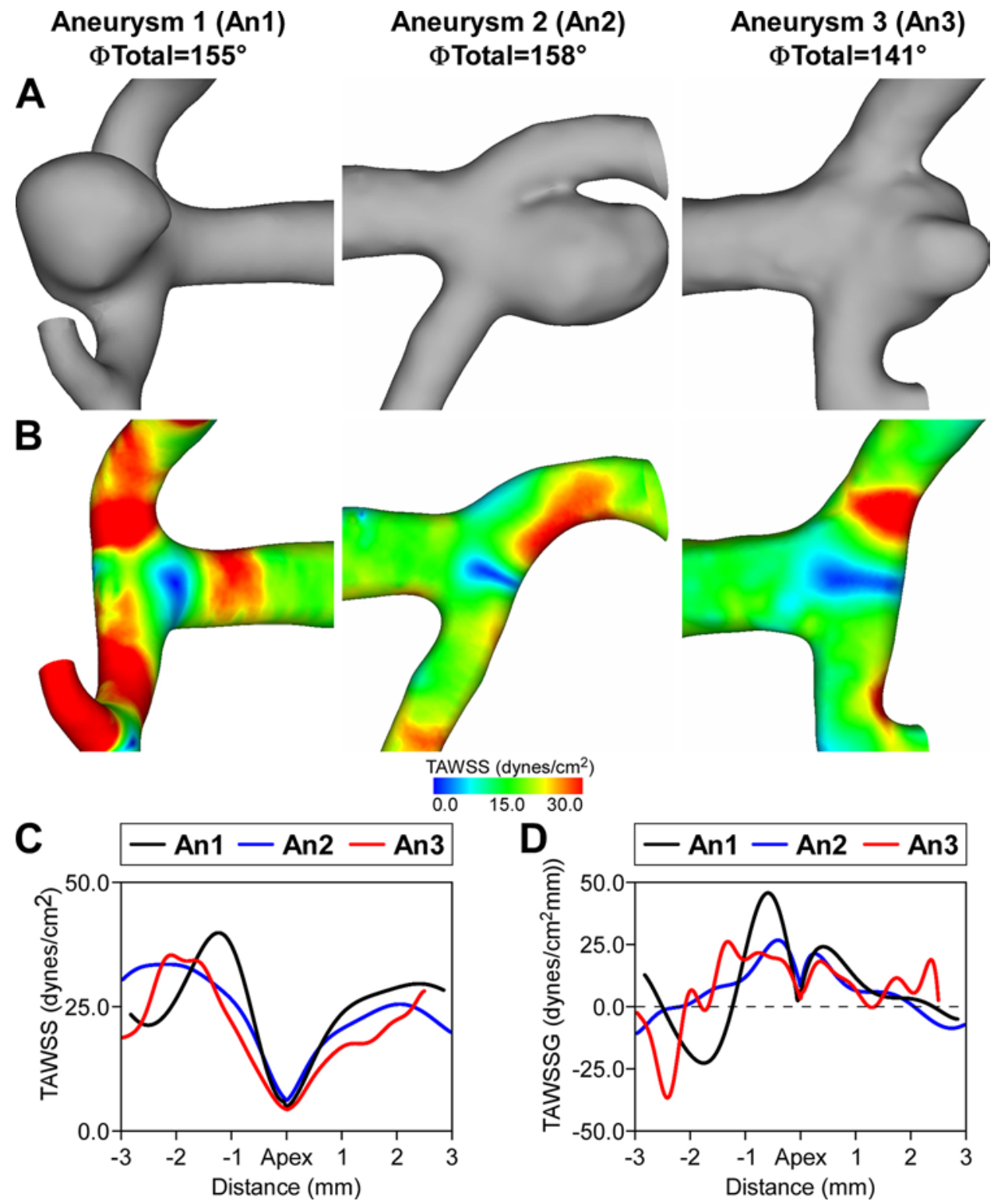

FIG. 7. CFD analysis of 3 patients with aneurysms at the MCA bifurcation. A: Patient-derived model prior to aneurysm removal using Laplacian smoothing. B: TAWSS. C: Plot of TAWSS on longitudinal 1D cut at the apex. D: Plot of TAWSSG on longitudinal $1 \mathrm{D}$ cut at the apex. Figure is available in color online only.

to become susceptible to sufficient biological change for aneurysm initiation to occur. ${ }^{10,18}$ Additional findings in patient data suggested that angles in healthy controls increased with age (especially after 35 years), and that they increased significantly more in women-which, together with the findings presented here, are consistent with older age and female sex representing additional risk factors for aneurysm formation. ${ }^{25}$

In contrast, asymmetrical bifurcations trigger positive WSSG on the side of the more obtusely angled daughter branch. The area of aneurysmogenic positive WSSG widens at increased angles. This could explain why the majority of bifurcation aneurysms occur off the daughter vessel associated with the larger angle. ${ }^{1}$ In fact, a threshold of $83^{\circ}$ was previously proposed for the daughter vessel angle in patient data, with wider angles being associated with aneurysm presence occurring off that particular branch. This correlates well with our simulations in asymmetrical bifurcations in which a widening of the negative WSSG area outside of the apex region occurs at a daughter angle of $90^{\circ}$.

In both symmetrical and asymmetrical bifurcations, the region exposed to positive WSSG increases at higher flow rates. Looking at the geometry of the inflow vessel, a curved proximal vessel triggers positive WSSG at the apex, on the side corresponding to the outer side of the curve, as the flow enters the bifurcation following the vessel's circle of curvature. For this type of geometry, in contrast to symmetrical and asymmetrical bifurcations on straight vessels, the impingement area migrates downstream toward the positive WSSG region. This displacement is maximal during the systolic phase and is exacerbated at high flow 
rates. This is relevant because in animal models the degree of destructive remodeling accounting for medial thinning and luminal bulging was correlated with the magnitude of the hemodynamic insult at the bifurcation. ${ }^{18}$ However, our research shows that high-flow conditions potentiate the effect of wider bifurcation geometry, but do not cause positive WSSG in narrow angles.

The potential clinical utility of screening high-risk patients based on bifurcation and vessel geometry remains to be investigated. It should be noted that angular remodeling resulting in narrower bifurcations was previously reported locally in aneurysmal bifurcations that underwent stentmediated coiling. ${ }^{8}$ Stent placement at bifurcations led to a significant biphasic angular modification (increase in the lateral angles between stented parent and daughter vessels, resulting in a decrease of the total bifurcation angle), effectively altering the bifurcation morphology and the local hemodynamic environment. ${ }^{9}$ This alteration of bifurcation geometry toward a narrower angle was reported in both distal and proximal bifurcation locations (including MCA). ${ }^{8}$ Even more dramatic remodeling toward bifurcation angle narrowing was reported in BA aneurysms treated with Y-stenting across bifurcations. ${ }^{9}$ The findings presented here suggest that mechanisms to decrease the total bifurcation angle could potentially mitigate conditions favorable to cell degradation and lower the risk for aneurysm initiation or recurrence.

\section{Study Limitations}

In the absence of patient-specific flow rates, this study used representative velocity and boundary conditions, as previously reported. ${ }^{12,14,25}$ It should be noted that in both parametric and patient-derived models, the inflow vessel was kept sufficiently long to enable the laminar flow to become fully developed and avoid entrance effects. In addition, the potential effect of the flow velocity on WSS and WSSG was evaluated by repeating the analysis using double the initial flow rate. It was encouraging that the increase in flow rate did not induce positive WSSG in narrow bifurcations. This suggests strongly that, whereas flow rate can potentiate the effect of the underlying geometry, it is the morphology of the bifurcation that determined the occurrence of aneurysmogenic high positive WSSG at the apex.

\section{Conclusions}

Local shear forces and spatial gradients at the bifurcation apex are highly dependent on the bifurcation and inflow vessel geometry. Whereas narrow bifurcations are characterized by mostly protective negative WSSG, wider and asymmetrical bifurcations, as well as bifurcations with curved inflow vessels, are exposed to aneurysmogenic positive WSSG, previously associated with destructive remodeling, indicative of aneurysm formation. A threshold angle of $85^{\circ}$ was established, above which bifurcation apices are exposed to aneurysmogenic positive WSSG conditions. The findings presented here correlate to results previously reported in patients in whom aneurysms occurred exclusively in bifurcations larger than $100^{\circ}$. Whether or not aneurysmogenic positive WSSG oc- curs at the apex appears to be a function of parent vessel and bifurcation geometry, and not of flow rate, although the latter can affect the extent of the affected apical area.

\section{References}

1. Baharoglu MI, Lauric A, Safain MG, Hippelheuser J, Wu C, Malek AM: Widening and high inclination of the middle cerebral artery bifurcation are associated with presence of aneurysms. Stroke 45:2649-2655, 2014

2. Bowker TJ, Watton PN, Summers PE, Byrne JV, Ventikos Y: Rest versus exercise hemodynamics for middle cerebral artery aneurysms: a computational study. AJNR Am J Neuroradiol 31:317-323, 2010

3. Cebral JR, Sheridan M, Putman CM: Hemodynamics and bleb formation in intracranial aneurysms. AJNR Am J Neuroradiol 31:304-310, 2010

4. DePaola N, Gimbrone MA Jr, Davies PF, Dewey CF Jr: Vascular endothelium responds to fluid shear stress gradients. Arterioscler Thromb 12:1254-1257, 1992

5. Dolan JM, Kolega J, Meng H: High wall shear stress and spatial gradients in vascular pathology: a review. Ann Biomed Eng 41:1411-1427, 2013

6. Dolan JM, Meng H, Singh S, Paluch R, Kolega J: High fluid shear stress and spatial shear stress gradients affect endothelial proliferation, survival, and alignment. Ann Biomed Eng 39:1620-1631, 2011

7. Finlay HM, Whittaker P, Canham PB: Collagen organization in the branching region of human brain arteries. Stroke 29:1595-1601, 1998

8. Gao B, Baharoglu MI, Cohen AD, Malek AM: Stent-assisted coiling of intracranial bifurcation aneurysms leads to immediate and delayed intracranial vascular angle remodeling. AJNR Am J Neuroradiol 33:649-654, 2012

9. Gao B, Baharoglu MI, Cohen AD, Malek AM: Y-stent coiling of basilar bifurcation aneurysms induces a dynamic angular vascular remodeling with alteration of the apical wall shear stress pattern. Neurosurgery 72:617-629, 2013

10. Gao L, Hoi Y, Swartz DD, Kolega J, Siddiqui A, Meng H: Nascent aneurysm formation at the basilar terminus induced by hemodynamics. Stroke 39:2085-2090, 2008

11. Hassan T, Timofeev EV, Saito T, Shimizu H, Ezura M, Matsumoto Y, et al: A proposed parent vessel geometry-based categorization of saccular intracranial aneurysms: computational flow dynamics analysis of the risk factors for lesion rupture. J Neurosurg 103:662-680, 2005

12. Hippelheuser JE, Lauric A, Cohen AD, Malek AM: Realistic non-Newtonian viscosity modelling highlights hemodynamic differences between intracranial aneurysms with and without surface blebs. J Biomech 47:3695-3703, 2014

13. Kono K, Fujimoto T, Terada T: Proximal stenosis may induce initiation of cerebral aneurysms by increasing wall shear stress and wall shear stress gradient. Int J Numer Methods Biomed Eng 30:942-950, 2014

14. Lauric A, Hippelheuser J, Cohen AD, Kadasi LM, Malek AM: Wall shear stress association with rupture status in volume matched sidewall aneurysms. J Neurointerv Surg 6:466-473, 2014

15. Lauric A, Safain MG, Hippelheuser J, Malek AM: High curvature of the internal carotid artery is associated with the presence of intracranial aneurysms. J Neurointerv Surg 6:733-739, 2014

16. Malek AM, Alper SL, Izumo S: Hemodynamic shear stress and its role in atherosclerosis. JAMA 282:2035-2042, 1999

17. Meng H, Wang Z, Hoi Y, Gao L, Metaxa E, Swartz DD, et al: Complex hemodynamics at the apex of an arterial bifurcation induces vascular remodeling resembling cerebral aneurysm initiation. Stroke 38:1924-1931, 2007

18. Metaxa E, Tremmel M, Natarajan SK, Xiang J, Paluch RA, 
Mandelbaum M, et al: Characterization of critical hemodynamics contributing to aneurysmal remodeling at the basilar terminus in a rabbit model. Stroke 41:1774-1782, 2010

19. Nagel T, Resnick N, Dewey CF Jr, Gimbrone MA Jr: Vascular endothelial cells respond to spatial gradients in fluid shear stress by enhanced activation of transcription factors. Arterioscler Thromb Vasc Biol 19:1825-1834, 1999

20. Nixon AM, Gunel M, Sumpio BE: The critical role of hemodynamics in the development of cerebral vascular disease. J Neurosurg 112:1240-1253, 2010

21. Rossitti S, Löfgren J: Vascular dimensions of the cerebral arteries follow the principle of minimum work. Stroke 24:371-377, 1993

22. Schirmer CM, Malek AM: Wall shear stress gradient analysis within an idealized stenosis using non-Newtonian flow. Neurosurgery 61:853-864, 2007

23. Sforza DM, Putman CM, Cebral JR: Hemodynamics of cerebral aneurysms. Annu Rev Fluid Mech 41:91-107, 2009

24. Szymanski MP, Metaxa E, Meng H, Kolega J: Endothelial cell layer subjected to impinging flow mimicking the apex of an arterial bifurcation. Ann Biomed Eng 36:1681-1689, 2008

25. Tütüncü F, Schimansky S, Baharoglu MI, Gao B, Calnan
D, Hippelheuser J, et al: Widening of the basilar bifurcation angle: association with presence of intracranial aneurysm, age, and female sex. J Neurosurg 121:1401-1410, 2014

\section{Disclosures}

Dr. Malek has received research funding from Stryker Neurovascular for research that is unrelated to the submitted work. He also has direct stock ownership in CereVasc LLC, and is a speaker for Microvention-Terump.

\section{Author Contributions}

Conception and design: Malek. Acquisition of data: Hippelheuser. Analysis and interpretation of data: Malek, Hippelheuser. Drafting the article: Lauric. Critically revising the article: Malek, Lauric. Approved the final version of the manuscript on behalf of all authors: Malek. Administrative/technical/material support: Malek, Lauric. Study supervision: Malek.

\section{Correspondence}

Adel M. Malek: Tufts Medical Center, Boston, MA. amalek@ tuftsmedicalcenter.org. 